\title{
Grazing Incidence Neutron Diffraction from Large Scale 2D Structures
}

B.P. Toperverg ${ }^{1,3}$, G.P. Felcher ${ }^{2}$, V.V. Metlushko ${ }^{2}$, V. Leiner ${ }^{4}$, R. Siebrecht ${ }^{4}$, O. Nikonov ${ }^{1,5}$

${ }^{1}$ Institut Laue - Langevin, B.P.156, 38042 Grenoble Cedex 9 France

${ }^{2}$ Argonne National Laboratory, 9700 S. Cass Ave., Argonne, IL 60439 USA

${ }^{3}$ Petersburg Nuclear Physics Institute, Gatchina, St. Petersburg 188350, Russia

${ }^{4}$ Institut für Experimetalphysik IV, Ruhr-Universität, D-44780 Bochum, Germany

${ }^{5}$ Joint Institute for Nuclear Research, 141980 Dubna, Moscow Region, Russia

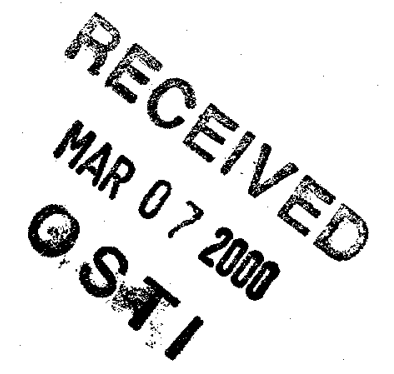

$6^{\text {th }}$ Conference on Scattering of X-rays and Neutrons from Surfaces, Noordwij, Netherlands, October 8, 1999, to be published in Physica B

\begin{abstract}
The submitted manuscript has been created by the University of Chicago as Operator of Argonne National Laboratory ("Argonne") under Contract No. W-31-109-ENG-38 with the U.S. Department of Energy. The U.S. Government retains for itself, and others acting on its behalf, a paid-up, nonexclusive, irrevocable worldwide license in said article to reproduce, prepare derivative works, distribute copies to the public, and perform publicly and display publicly, by or on behalf of the Government.
\end{abstract}

This work was supported by the U.S. Department of Energy, Office of Basic Energy Sciences Division of Materials Sciences, under contract \#W-31-109-ENG-38, Russian State Program "Neutron Research of Condensed Matter" and RFFI-Grant No. L-EN-96-15-96775. 


\section{DISCLAIMER}

This report was prepared as an account of work sponsored by an agency of the United States Government. Neither the United States Government nor any agency thereof, nor any of their employees, make any warranty, express or implied, or assumes any legal liability or responsibility for the accuracy, completeness, or usefulness of any information, apparatus, product, or process disclosed, or represents that its use would not infringe privately owned rights. Reference herein to any specific commercial product, process, or service by trade name, trademark, manufacturer, or otherwise does not necessarily constitute or imply its endorsement, recommendation, or favoring by the United States Government or any agency thereof. The views and opinions of authors expressed herein do not necessarily state or reflect those of the United States Government or any agency thereof. 


\section{DISCLAIMER}

Portions of this document may be illegible in electronic image products. Images are produced from the best available original document. 


\title{
Grazing Incidence Neutron Diffraction from Large Scale 2D Structures
}

\author{
B.P. Toperverg ${ }^{1,3}$, G.P. Felcher ${ }^{2}$, V.V. Metlushko ${ }^{2}$, V. Leiner ${ }^{4}$, R. Siebrecht ${ }^{4}$, O. Nikonov ${ }^{1,5}$ \\ ${ }^{1}$ Institut Laue - Langevin, B.P.156, 38042 Grenoble Cedex 9, France \\ ${ }^{2}$ Argonne National Laboratory, 9700 South Cass Avenue, Argonne, IL, USA \\ ${ }^{3}$ Petersburg Nuclear Physics Institute, Gatchina, St.Petersburg 188350, Russia \\ ${ }^{4}$ Institut für Experimentalphysik IV, Ruhr-Universität, D-44780 Bochum, Germany \\ ${ }^{5}$ Joint Instutute for Nuclear Research, 141980 Dubna, Moscow region, Russia
}

(October 30, 1999)

\begin{abstract}
The distorted wave Born approximation (DWBA) is applied to evaluate the diffraction pattern of neutrons (or X-rays) from a 2D array of dots deposited onto a dissimilar substrate. With the radiation impinging on the surface at a grazing incidence angle $\alpha$, the intensities diffracted both in and out the plane of specular reflection are calculated as a function of the periodicity of the array, height and diameter of the dots. The results are presented in the form of diffracted intensity contours in a plane with coordinates $\alpha$ and $\alpha^{\prime}$, the latter being the glancing angle of scattering. The optimization of the experimental conditions for polarized neutron experiments on submicron dots is discussed. The feasibility of such measurements is confirmed by a test experiment.

Keywords: Neutron diffraction, Dot array, Grazing incidence

61.12.Ex,75.70.Ak,75.70.Cn
\end{abstract}

Typeset using REVTEX 


\section{DIFFRACTION GEOMETRY}

In the last few years it has become possible to produce periodic arrays of submicron "dots" [1-3] and nano-scale [4,5], two dimensional (2D) structures on flat surfaces or embedded in metallic, semiconducting or superconducting films and multilayers. High precision control of the size, period and symmetry of the micro and nano structures enables the fabrication of systems of great interest for optical and magnetic storage applications. In order to check the fabrication parameters a number of different techniques, such as optical, electron and atomic force microscopy, as well as optical and electron diffraction are widely employed. Recently, the magnetic properties of a 2D lattice of holes (antidots) in a Fe film have been studied [6] by means of magneto-optic Kerr effect in combination with Brillouin scattering and magnetic force microscopy. However, these methods can hardly be useful if the structure is buried under a non transparent film. If the film is not too thick grazing incidence diffraction (GID) of X-rays [7] may deliver rather complete information on the dot arrangement, while the use of polarized neutron GID is most suited to study the magnetic structures implanted into thick films. In the present paper the equations for GID from 2D artificial structure will be derived and analyzed with the aim of optimizing forthcoming experiments.

Conventionally it is assumed that the optimal conditions for the diffraction on a lattice are satisfied if the wavelength $\lambda$ of X-rays or neutrons is scaled with the reciprocal lattice spacing $a$. Therefore, at first sight it seems that neutron or X -ray diffractometry is not the proper choice to study a lattice with a spacing $a$ of the order of microns. This statement, however does not always hold for the case of $2 \mathrm{D}$ structures on a flat surface when viewed at grazing incidence. Let us consider the case in which the incoming radiation is at a grazing angle $\alpha$ with respect to the basic plane and the $2 \mathrm{D}$ structure is defined by reciprocal lattice vectors $\tau$.

If $\kappa, \kappa^{\prime}$ are the wave vector projections of the incoming and scattered beams onto the basal plane, $\mathrm{Q}_{\|}=\boldsymbol{\kappa}^{\prime}-\kappa$ is the lateral wave vector transfer, and $\omega$ is the in-plane scattering angle between the vectors $\kappa$ and $\kappa^{\prime}$, then the Bragg law $\mathbf{Q}_{\|}=\boldsymbol{\tau}$ is fulfilled at $\alpha^{\prime}=\alpha \boldsymbol{\tau}$ and 
$\omega=\omega_{\tau}$, where

$$
\begin{gathered}
\cos ^{2} \alpha_{\tau}=\cos ^{2} \alpha+2 \Theta_{\tau} \cos \chi_{\tau} \cos \alpha+\Theta_{\tau}^{2} \\
\sin \omega_{\tau} \cos \alpha_{\tau}=\Theta_{\tau} \sin \chi_{\tau}
\end{gathered}
$$

$\chi \tau$ is the angle between the directions of $\kappa$ and the vector $\tau$ (see Fig.1), and $\Theta_{\tau}=\tau / k$. At $\lambda \ll a$ the amplitudes of the Bragg peaks may have appreciable values only at $\alpha \ll 1$ and $\alpha^{\prime} \ll 1$, and then:

$$
\begin{gathered}
\alpha_{\tau} \approx \sqrt{\alpha^{2}-2 \Theta_{\tau} \cos \chi_{\tau}} \ll 1, \\
\omega_{\tau} \approx \Theta_{\tau} \sin \chi_{\tau} \ll 1
\end{gathered}
$$

where $\Theta_{\tau}=\tau / k$. As these equations determine the peak positions as functions of $\alpha$ and $\alpha^{\prime}$, the scattered intensity may conveniently be represented as a contour plot in the $\left\{\alpha^{\prime}, \alpha\right\}-$ plane. Let us for instance consider a square lattice (Fig.1) in the symmetrical case in which the basic reciprocal vector $\tau_{10}$ is directed along $\kappa$, and thus, $\omega_{10}=\omega_{\overline{1} 0}=0$. Then the representation of the equation $\alpha^{2}=\alpha_{\tau}^{2}(\alpha)$ in $\left\{\alpha, \alpha^{\prime}\right\}$-plane is that of on hyperbola cutting the $\alpha$ axis at $\sqrt{2 \Theta_{1}}$ : neutrons incident at smaller values of $\alpha$ are unable to excite this diffraction lines. Similarly, $\tau_{\overline{1} 0}$ gives rise at a diffraction line at loci defined by an hyperbolas that cuts the $\alpha^{\prime}$ axis at $\pm \sqrt{2 \Theta_{1}}$.

The diffraction effects of the reciprocal vectors $\tau_{01}$ and $\tau_{0 \overline{1}}$ are significantly different. For $\omega= \pm \Theta_{1}$ the diffracted beams loci in $\left\{\alpha \alpha^{\prime}\right\}$-plane are coincident with the reflected or transmitted beam: this is because they occure for a modulation of the in-plane potential exactly perpendicular to the incoming wave. However, turning the reciprocal lattice into asymmetric position has dramatic effect on the Bragg peaks at $\pm \alpha_{01}$ and $\pm \alpha_{0 \overline{1}}$. The degeneracy for these Bragg lines is lifted: then one can, in principle, observe all four peaks corresponding to the curves $\alpha^{2}-\alpha^{2}= \pm 2 \Theta_{1} \chi$. In Fig.2 is presented an example, when $\chi=0.4^{\circ}$.

The example shown indicates that the Bragg law $Q_{\|}=\tau$ defines not only the in-plane scattering angle $\omega$, but also the angle $\alpha^{\prime} \neq \alpha$ between the outgoing wave vector and the 
basic $2 \mathrm{D}$ plane. In this geometry small lateral momentum transfers result in appreciable changes in $Q_{\perp}$ : for instance, a resolution in $Q_{\perp}$ of $\sim 10^{-3} \AA$ provides a sensitivity to $Q_{\|}$ down to $10^{-6} \AA^{-1}$, a range well matched with lattice spacings of the order of microns. We want to calculate now the intensities expected for the diffraction from such a $2 \mathrm{D}$ structure.

\section{SCATTERING AMPLITUDE}

Let us consider a set of ferromagnetic particles forming a periodic $2 \mathrm{D}$ lattice in the $\mathrm{XY}$-plane. For instance, the particles may have height $d$ and be deposited on a thick flat substrate. The neutron wave incident on such a sample is reflected and refracted by the optical potential of the substrate, and also diffracted by the lattice. If the angle of incidence is low, the optical effect should be accounted for exactly. However, as the diffracted intensity is usually weak its effect can be regarded as a perturbation. The potential $\mathcal{V}(\mathbf{r})$ representing the neutron interaction with the system may be decomposed into a sum

$$
\mathcal{V}(\mathbf{r})=\mathcal{V}_{0}(z)+\mathcal{V}_{d}(\mathbf{r})
$$

where the first term depends solely on the coordinate $z$ normal to the surface and may be approximated by the optical potential: $\mathcal{V}_{0}(z)=0$ at $z<0$, and $\mathcal{V}_{0}=\hbar^{2} p_{s}^{2} / 2 m$ in the substrate. Here $p_{s}^{2}=4 \pi n_{s} b_{s} ; p_{s}$ is the critical wave number of the total reflection and $n_{s} b_{s}$ is the neutron scattering length density (SLD) for the substrate.

The second term in the Eq.(5) is due to the dots with SLD $n_{d} b_{d}$, and can be written in the form:

$$
\mathcal{V}_{d}(\mathbf{r})=\frac{\hbar^{2} p_{d}^{2}}{2 m} \sum_{i} F\left(\boldsymbol{\rho}-\rho_{i}, z-d\right),
$$

where $p_{d}^{2}=4 \pi n_{d} b_{d}, i$ is a running index which indicates the particle position on the substrate surface, and $F(\rho, z)$ is the particle shape function.

Since $\mathcal{V}_{d}(\mathbf{r})$ depends on the $3 \mathrm{D}$ coordinate $\mathbf{r}=\{\boldsymbol{\rho}, z\}$, it causes diffraction in off-specular directions. Within the first order of the distorted wave Born approximation (DWBA) the diffraction amplitude $f\left(\mathbf{k}^{\prime}, \mathbf{k}\right)$ is proportional to the matrix element of the potential $\mathcal{V}_{d}(\mathbf{r})$ 


$$
f\left(\mathbf{k}^{\prime}, \mathbf{k}\right)=-\frac{m}{2 \pi \hbar^{2}} \int d \mathbf{r} \tilde{\Psi}\left(\mathbf{k}^{\prime}, \mathbf{r}\right) \mathcal{V}_{d}(\mathbf{r}) \Psi(\mathbf{k}, \mathbf{r}),
$$

where the wave functions $\tilde{\Psi}\left(\mathbf{k}^{\prime}, \mathbf{r}\right)$ and $\Psi(\mathbf{k}, \mathbf{r})$ are the exact solutions for the potential $\mathcal{V}_{0}$ with the proper initial and boundary conditions. Thus in free space $\Psi(\mathbf{k}, \mathbf{r})$ is a plane wave approaching the sample surface from the neutron source, while $\tilde{\Psi}\left(\mathbf{k}^{\prime}, \mathbf{r}\right)$ is a wave approaching the sample in the direction opposite to the direction of observation: $\Psi(\mathbf{k}, \mathbf{r})=e^{i \kappa \boldsymbol{\rho}} \psi\left(p_{0}, z\right)$ and $\widetilde{\Psi}\left(\mathbf{k}^{\prime}, \mathbf{r}\right)=e^{-i \kappa^{\prime}} \boldsymbol{\rho} \widetilde{\psi}\left(p_{0}^{\prime}, z\right)$, where $p_{0}=k \sin \alpha, p_{0}^{\prime}=k \sin \alpha^{\prime}$ are perpendicular to the surface components of $\mathbf{k}, \mathbf{k}^{\prime}, \psi\left(p_{0}, z\right)$ and $\tilde{\psi}\left(p_{0}^{\prime}, z\right)$ are the exact solutions of the one dimensional Schrödinger equation with the potential $\mathcal{V}_{0}(z)$. Due to the factorization of the wave functions,

$$
f\left(\mathbf{Q}_{\|}, p_{0}^{\prime}, p_{0}\right)=-\frac{p_{d}^{2}}{4 \pi} \sum_{i} e^{i \mathbf{Q}_{\|} \rho_{i}} F\left(\mathbf{Q}_{\|} ; p_{0}^{\prime}, p_{0}\right),
$$

where $F\left(\mathbf{Q}_{\|} ; p_{0}^{\prime}, p_{0}\right)$ is the matrix element:

$$
F\left(\mathbf{Q}_{\|} ; p_{0}^{\prime}, p_{0}\right)=\int d z \tilde{\psi}\left(p_{0}^{\prime}, z\right) F\left(\mathbf{Q}_{\|}, z\right) \psi\left(p_{0}, z\right),
$$

and $F\left(\mathrm{Q}_{\|}, z\right)$ is the 2D Fourier transform of the scatterer shape function $F(\boldsymbol{\rho}, z)$. In the case the scatterers have columnar character $F\left(\mathbf{Q}_{\|} ; p_{0}^{\prime}, p_{0}\right)=F_{\|}\left(\mathbf{Q}_{\|}\right) F_{\perp}\left(p_{0}^{\prime}, p_{0}\right)$, with

$$
F_{\perp}\left(p_{0}^{\prime}, p_{0}\right)=\int_{-d}^{0} d z \widetilde{\psi}\left(p_{0}^{\prime}, z\right) \psi\left(p_{0}, z\right)
$$

$F_{\|}\left(\mathbf{Q}_{\|}, z\right)$ depends on the actual cross section of the column, and $d$ is the column height.

The wave functions $\psi\left(p_{0}, z\right)$ and $\widetilde{\psi}\left(p_{0}^{\prime}, z\right)$ are linear combinations of plane waves incident and reflected from the substrate: $\psi\left(p_{0}, z\right)=e^{i p_{0} z}+r e^{-i p_{0} z}$, where $r=\left(p-p_{0}\right) /\left(p+p_{0}\right)$ is the Fresnel reflectance, $p=\sqrt{p_{0}^{2}-p_{s}^{2}}$ is the neutron wave number within the substrate. If scattering occurs in the reflection hemisphere, i.e. above the sample "horizon", then the wave function $\tilde{\psi}\left(p_{0}^{\prime}, z\right)$ has exactly the same form as given by Eqs.(11) with the argument $p_{0}$ substituted by $p_{0}^{\prime}$. The explicit equation for $F_{\perp}\left(p_{0}^{\prime}, p_{0}\right)$ is

$$
F_{\perp}\left(p_{0}^{\prime}, p_{0}\right)=F_{+}^{*}+r^{\prime} F_{-}^{*}+r F_{-}+r^{\prime} r F_{+} \text {. }
$$

This equation contains four terms, and each of them corresponds to one of the possible scattering processes of incident or reflected waves. The "transverse" form-factors are: 


$$
\begin{aligned}
& F_{+}=\frac{\exp \left[i\left(p_{0}^{\prime}+p_{0}\right) d\right]-1}{i\left(p_{0}^{\prime}+p_{0}\right)} \\
& F_{-}=\frac{\exp \left[i\left(p_{0}^{\prime}-p_{0}\right) d\right]-1}{i\left(p_{0}^{\prime}-p_{0}\right)}
\end{aligned}
$$

where $r^{\prime}=r\left(p_{0}^{\prime}\right)$.

It is important to note that the "transverse" form-factor $F_{\perp}\left(p_{0}^{\prime}, p_{0}\right)$ is a function of two variables, i.e. the normal to the surface components $p_{0}^{\prime}$ and $p_{0}$ of the outgoing and incoming wave vector, rather than the transverse component of the wave vector transfer $Q_{\perp}=p_{0}^{\prime}+p_{0}$. The latter variable is the proper choice only in the Born (kinematic) approximation, which is valid for values of $p_{0}^{\prime}$ and $p_{0}$ far beyond the range of the total reflection. In the kinematic approximation $p^{\prime} \approx p_{0}^{\prime}, p \approx p_{0}$, all reflected waves are neglected, and the transverse form factor takes the more easily recognizable form

$$
F_{\perp}\left(Q_{\perp}\right) \approx e^{i Q \perp d / 2} \frac{\sin \left(Q_{\perp} d / 2\right)}{Q_{\perp} / 2}
$$

which at low angles depends only upon the scattering angle $\theta=\alpha^{\prime}+\alpha$. However, even in

this simplest case the total form-factor $F\left(\mathbf{Q}_{\|} ; p_{0}^{\prime}, p_{0}\right)$ lineshape is a function not only of the scattering angle $\theta$, but also of the angle of incidence: it may show a maximum not only at $\theta=0$, but also at $\theta \approx 2 \alpha$, which corresponds to "specular reflection" from the disc faces.

\section{AN EXAMPLE: FE DOTS ON SI}

In accordance with Eq.(4) the equation for the differential scattering cross section reads:

$$
\begin{gathered}
\frac{d \sigma}{d \Omega}=\frac{1}{4} \mathcal{N} p_{d}^{4} \mathcal{S}\left(\mathbf{Q}_{\|}\right)\left|F\left(\mathbf{Q}_{\|} ; p_{0}^{\prime}, p_{0}\right)\right|^{2} \\
\mathcal{S}\left(\mathbf{Q}_{\|}\right)=\left(1 / s_{0}\right) \sum \boldsymbol{\tau} \delta\left(\mathbf{Q}_{\|}-\boldsymbol{\tau}\right)
\end{gathered}
$$

where $\mathcal{N}$ is the number of scatterers illuminated by the incoming beam, and $s_{0}$ is the unit cell area. For cylindrical particles the form-factor

$$
\left|F\left(\mathbf{Q}_{\|} ; p_{0}^{\prime}, p_{0}\right)\right|^{2}=F_{\|}^{2}\left(Q_{\|}\right)\left|\sum_{\mu \nu} F_{\perp}^{\mu \nu}\left(p_{0}^{\prime}, p_{0}\right)\right|^{2}
$$

with $F_{\perp}^{\mu \nu}\left(p_{0}^{\prime}, p_{0}\right)$ given by Eqs.(11-13), and 


$$
F_{\|}\left(\mathbf{Q}_{\|}\right)=\frac{\pi \rho_{0}}{Q_{\|}} J_{1}\left(\rho_{0} Q_{\|}\right),
$$

for an array of cylindrical particles of radius $\rho_{0}$, corresponding to a particle shape function $F(\rho, z)=1$ at $\rho \leq \rho_{0}$ and $1 \leq z \leq d$, and zero otherwise.

In terms of the angles of incidence and scattering the conditions of occurrence of the Bragg reflection can be expressed as

$$
\begin{gathered}
\delta\left(\mathbf{Q}_{\|}-\boldsymbol{\tau}\right)=\frac{2}{k^{2}\left|\sin 2 \alpha^{\prime}\right|} \delta\left(\omega-\omega_{\boldsymbol{\tau}}\right) \\
{\left[\delta\left(\alpha^{\prime}-\alpha_{\boldsymbol{\tau}}\right)+\delta\left(\alpha^{\prime}+\alpha \boldsymbol{\tau}\right)\right]}
\end{gathered}
$$

In estimating the Bragg peak positions in the $\left\{\alpha^{\prime}, \alpha\right\}$ plane it was assumed that the equation for azimuthal angles $\omega=\omega_{\tau} \approx \Theta_{\tau} \chi_{\tau}$ is satisfied for each of the discussed reciprocal vectors $\tau$. Usually this is satisfied automatically because reflectometers have poor resolution with respect to the azimuthal angles, and all the Bragg lines for $\omega$ 's in the range of interest are just summed up. In accordance with Eq.(19) the form factor $F_{\|}(\tau) \approx \pi \rho_{0}^{2}$ at $\tau \rho_{0} \leq 1$, and it decays as $\tau^{-3 / 2}$ at $Q_{\|} \rho_{0} \gg 1$. Therefore this sum is well convergent and approaches the summation of $\omega_{\tau}$ to infinite limits. However, only relatively small number $n \sim a / \rho_{0}$ of terms bring appreciable contribution to this sum and small factor $\left(4 \pi^{2} \lambda^{2} / s_{0} \alpha\right) \ll 1$ in Eq.(20) is not compensated: the diffraction effect remains quite weak even at $\chi_{\tau} \ll 1$. At higher angles $\alpha^{\prime}, \alpha$ and/or $\chi_{\tau}$ the Bragg diffraction is additionaly suppressed by the transverse form factor given in Eq.(12).

Numerical calculations were made for the diffraction pattern of square $1 \times 1 \mu \mathrm{m}^{2}$ lattice of iron dots with diameter $3000 \AA$ and height $800 \AA$ deposited on a $\mathrm{SiO}_{2}$ wafer. Neutrons have a wave length of $\lambda=4.41 \AA$, and fall on the dot pattern at an angle $\chi=0.4^{\circ}$ from the lattice edge. For SLD were taken: for $\mathrm{SiO}_{2} n_{s} b_{s} \approx 03.9 \times 10^{-6} \AA^{-2}$, for iron $n_{d} b_{d} \approx 13 \times 10^{-6} \AA^{-2}$, as expected for fully magnetized iron and for neutrons polarized parallel to the direction of magnetization. With a resolution $\Delta \theta=0.02^{\circ}$, we find that the maximum intensity of the $(0, \pm 1)$ peaks may amount up to $2 \%$ of the total reflection. Much lower is the intensity of the $( \pm 1,0)$ reflections, which is suppresed by the form factor 
$F_{\perp}\left(p_{0}^{\prime}, p_{0}\right)$ at $p_{0}^{\prime}, p_{0} \geq d^{-1}$. The results of calculations are presented in Fig.2a, while in order to experimental results are depicted in Figs.3b. The experiment carried out at the polarized neutron reflectometer ADAM [8] at the Institut Laue-Langevin (Grenoble) shows that indeed the diffraction experiments on submicron size dot systems are feasible. However, the amount of intensity diffracted is such that such experiments can provide a satisfactory amount of physical information only for very selective problems and systems with suffisiently large area.

*Work is supported by US-DOE, BES-MS contract \#W-31-109-ENG-38, Russian State Program "Neutron Research of Condensed Matter" and RFFI-Grant No. L-EN-96-15-96775. 


\section{REFERENCES}

[1] J.I. Martin et al., Phys. Rev. Lett. 79, 1929 (1997).

[2] Y. Jaccard et al.; Phys. Rev. B 58, 8232 (1998).

[3] D.J. Morgan et al., Phys. Rev. Lett. 80, 3614 (1998).

[4] V. Holý et al., Phys. Rev. Lett. 83, 356 (1999).

[5] M. Grassi Alessi et al., Phys. Rev. B 59, 7620 (1999).

[6] P. Vavassori et al., Phys. Rev. . B 59, 6337 (1999).

[7] M.Tolan et al., Physica B 221 (1996) 53.

[8] A. Schreyer et al., Physica B 248 (1998) 349. 


\section{FIGURE CAPTIONS}

FIG.1 Geometry of the $(0,1)$ diffraction process from square lattice of dots.

FIG.2 a.) Schematic contour plots of the reflected and diffracted intensity as a function of the scattering angles $\alpha^{\prime}$ and the incidence angles $\alpha$; Sample is $1 \times 1 \mu \mathrm{m}^{2}$ lattice of iron dots with diameter $3000 \AA$ and height $800 \AA$ deposited onto $\mathrm{SiO}_{2}$ wafer, neutrons wave length $\lambda=4.41 \AA$, and the angle $\chi=0.4^{\circ}$,

b) experimental results for neutrons polarized along the dot magnetization. 


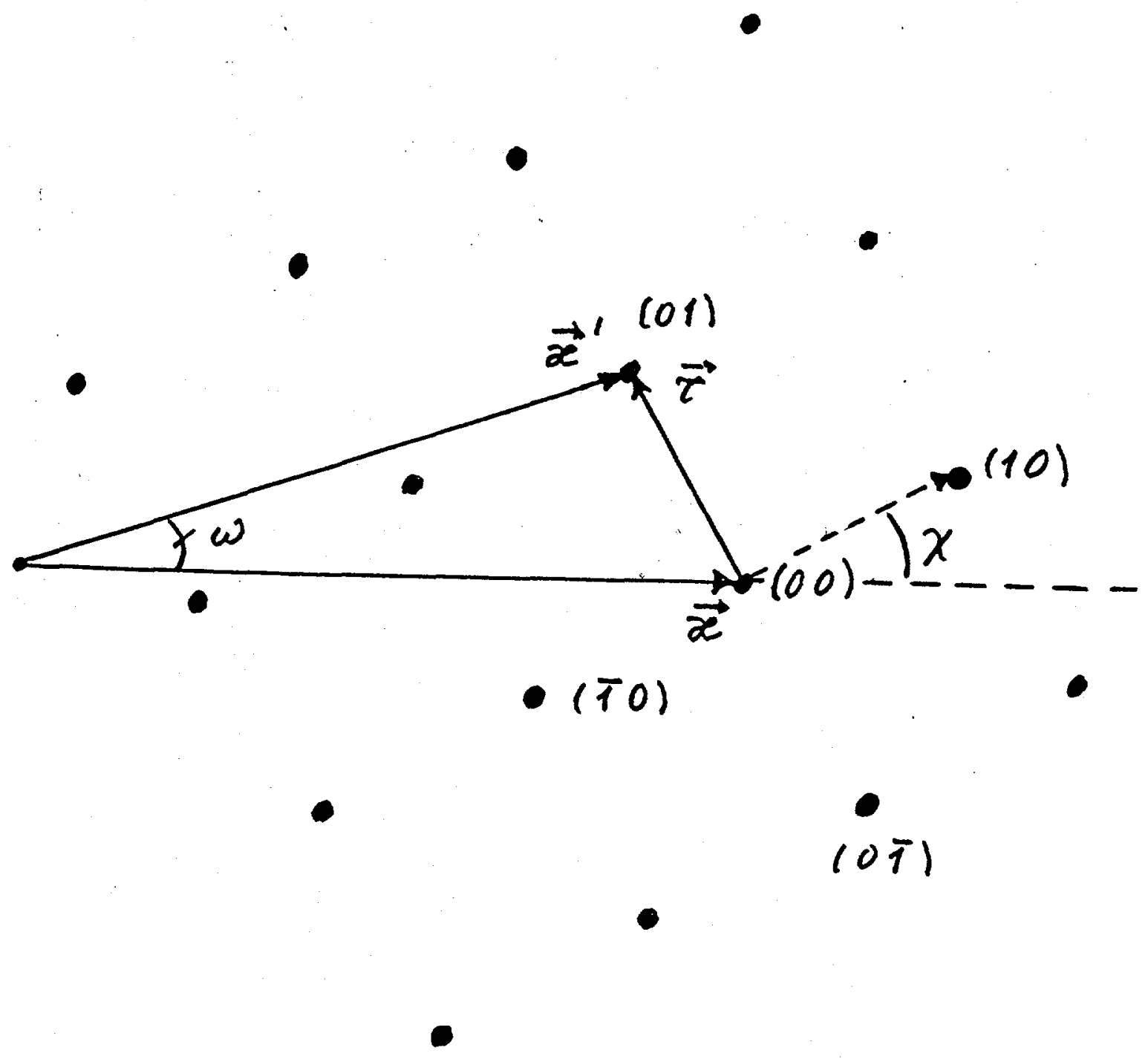

Fig. 1 


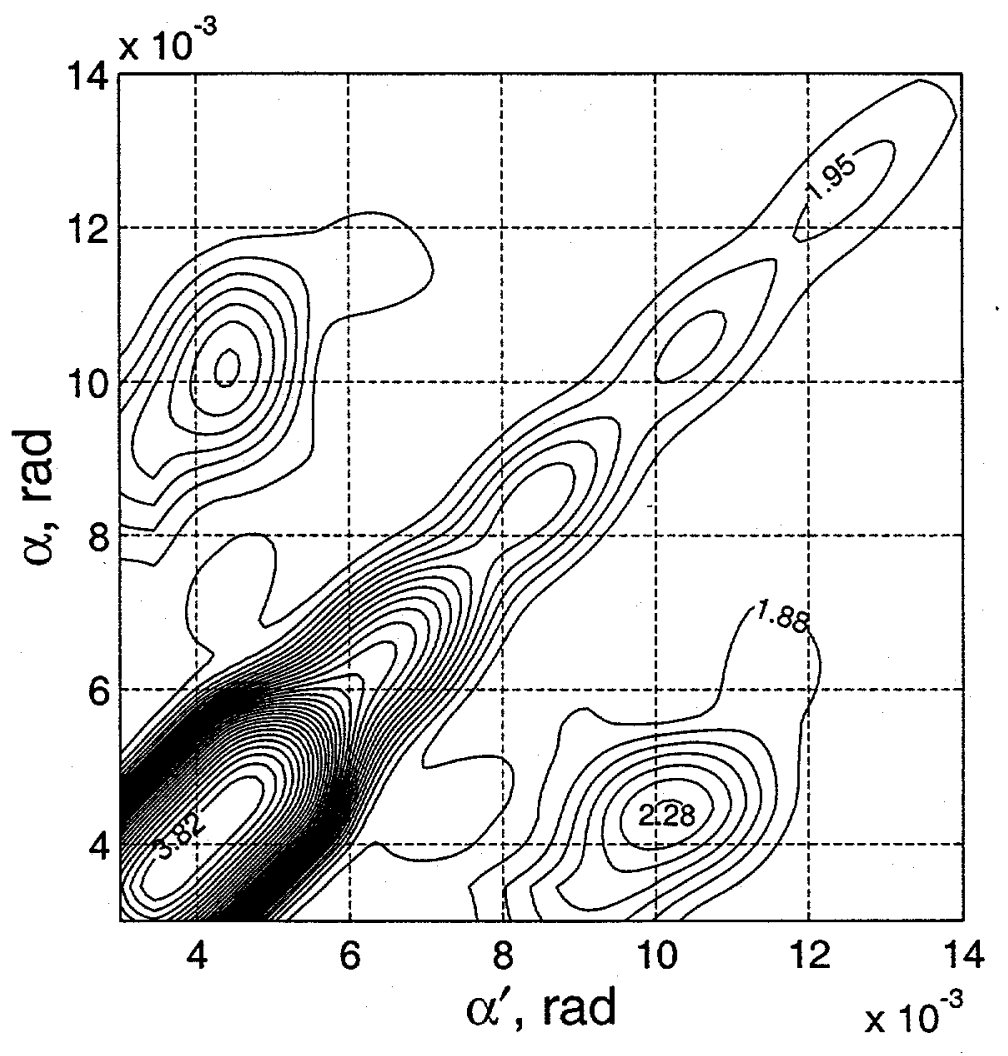

Fig. $2 a$ 


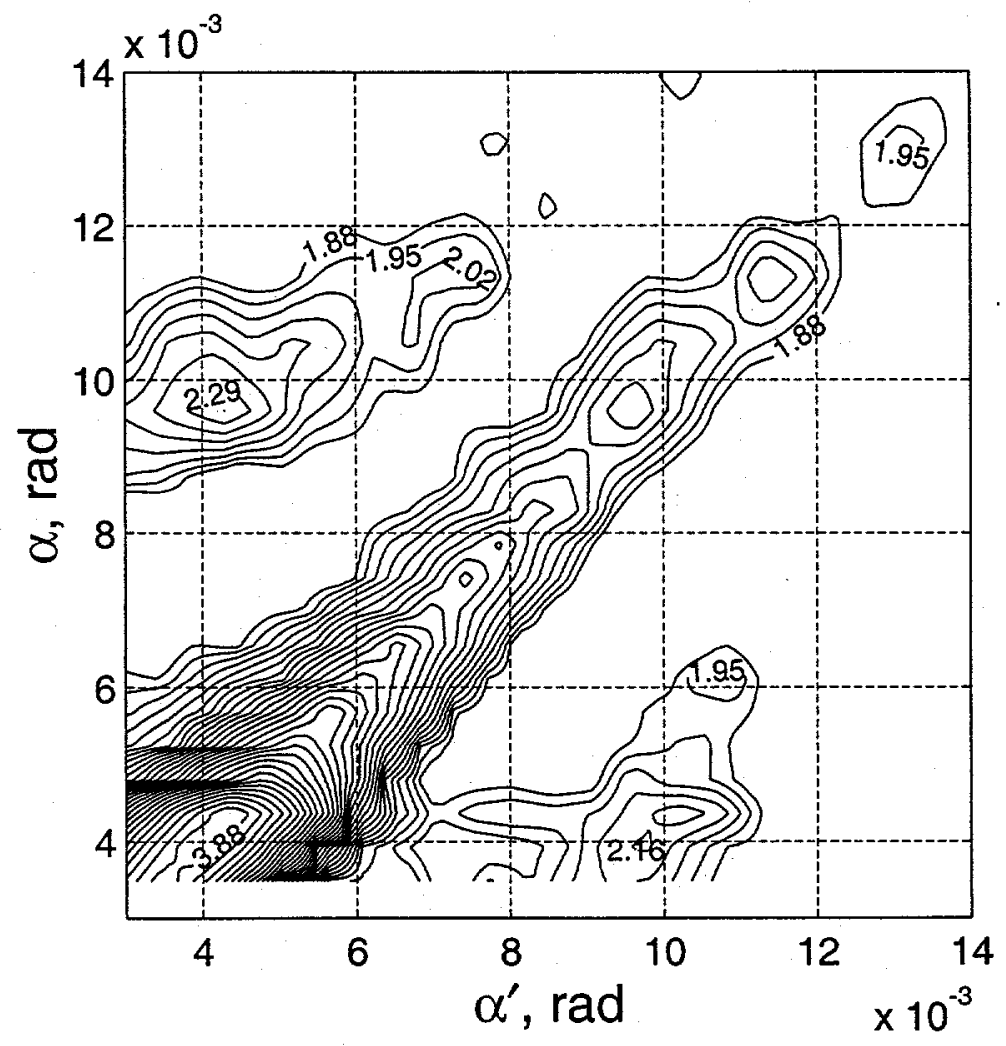

Fig 2.b 